\title{
Visual motion sensing onboard a 50-g helicopter flying freely under complex VICON-lighting conditions*
}

\author{
Franck Ruffier and Fabien Expert \\ Aix-Marseille University, Institute of Movement Science, Biorobotics Dept., 13288, Marseille, France \\ CNRS, UMR7287, 13288, Marseille, France
}

\begin{abstract}
In previous studies, we described how complicated tasks such as ground avoidance, terrain following, takeoff and landing can be performed using optic flow sensors mounted on a tethered flying robot called OCTAVE.

In the present study, a new programmable visual motion sensor connected to a lightweight Bluetooth module was mounted on a free-flying 50-gram helicopter called TwinCoax. This small helicopter model equipped with 3 IR-reflective markers was flown in a dedicated room equipped with a VICON system to record its trajectory. The results of this study show that despite the complex, adverse lighting conditions, the optic flow measured onboard matched the ground-truth optic flow generated by the free-flying helicopter's trajectory quite exactly.
\end{abstract}

Index Terms-Elementary motion detector (EMD), Optic flow, Micro-aerial vehicle (MAV), Free-flying Helicopter

\section{INTRODUCTION}

Several optic flow-based navigation systems have been developed during the past decade for use onboard terrestrial and aerial robots, fixed wing aircraft and medium-size helicopter models [1]-[13]. As far as we know, however, optic flow measurements obtained onboard a free-flying helicopter have never been compared so far with the ground-truth optic flow. Many methods of measuring the visual angular speed have been used for robotic purposes (see the Introduction to [14] for a review). These methods include those based on the "time of travel" scheme [15], [16], which was combined with off-the-shelf photodiodes and implemented on both a terrestrial robot [1] and several tethered flying robots [4], [17]. The performances of these visual motion sensors in terms of their resolution, accuracy, sensitivity, and invariance to contrast in real environments under a large range of illuminance values were recently assessed [14], [18] and favourably compared with those of off-the-shelf mouse motion sensors [19].

Although visual motion sensors are of great interest for robotic applications, very few attempts have been made so far to mount devices of this kind onboard miniature helicopters, especially under complex lighting conditions [2], [9]. Some authors have tested their camera-based optic flow

\footnotetext{
*This work was supported partly by CNRS Institutes (Life Science; Information Science; Engineering Science and Technology), by the AixMarseille University, by the French National Research Agency -ANR(EVA project under ANR-ContInt grant number: ANR-08-CORD-007-04) and by European Commission via the CURVACE project. The CURVACE project acknowledges the financial support of the Future and Emerging Technologies (FET) programme within the Seventh Framework Programme for Research of the European Commission, under FET-Open grant number: 237940 .
}

sensing technology on flying aerial vehicle, but the optic flow processing was performed offboard on a host computer (outdoor helicopter : [8], [12], indoor helicopter : [11], indoor ornithopter : [20]). Other authors have also started to use off-the-shelf computer mouse sensors as optic flow sensors onboard medium-size fixed-wing aircraft [5], [10] navigating outdoors.

The optic flow measurements $\left(\omega_{\text {median }}\right)$ made onboard a miniature helicopter flying freely over a natural landscape are compared here for the first time with the computed ground-truth optic flow $\left(\frac{v_{x}}{h}\right)$ generated by the aircraft's own trajectory under complex lighting conditions.

Section 2 gives a description of an optic-flow regulation scheme which was previously implemented on a tethered robot (OCTAVE) performing automatic landing, takeoff and terrain following. Section 3 describes the new programmable visual motion sensor incorporated in the free-flying helicopter named TwinCoAx. With this new bio-inspired 4-pixel motion sensor, programmable gains can be adjusted before the digitizing is performed in order to finely tune the visual sensor dynamic range for a broad range of illuminance. Section 4 focuses on the free-flight facilities, including the complex lighting conditions produced by the VICON system recording the free-flying helicopter's motion. Section 5 presents the 50-gram helicopter called TwinCoAx, which is equipped with a Bluetooth wireless communication link and a bio-inspired 4-pixel motion sensor. Section 6 shows the experimental results obtained, which confirm that our new programmable 4-pixel motion sensor was able to accurately measure the optic flow generated during the helicopter's freeflight despite the complex illuminance conditions pertaining in the experimental room and the onboard vibrations.

\section{BACKGROUND: OPTIC-FLOW REGULATION ON A PREVIOUS TETHERED HELICOPTER}

After carefully studying the ethological literature, we came up with an autopilot called OCTAVE (standing for Optical altitude Control sysTem for Autonomous Vehicles), which makes few demands in terms of its electronic implementation and may be just as appropriate for describing what occurs in insects as it is for controlling aircraft [3], [7]. A ventral OF sensor was integrated into a feedback loop driving the robot's lift, and thus the ground height, so as to compensate for any deviations of the OF sensor's output from a given set point [3], [4], [7], [22]. This simple OCTAVE autopilot 


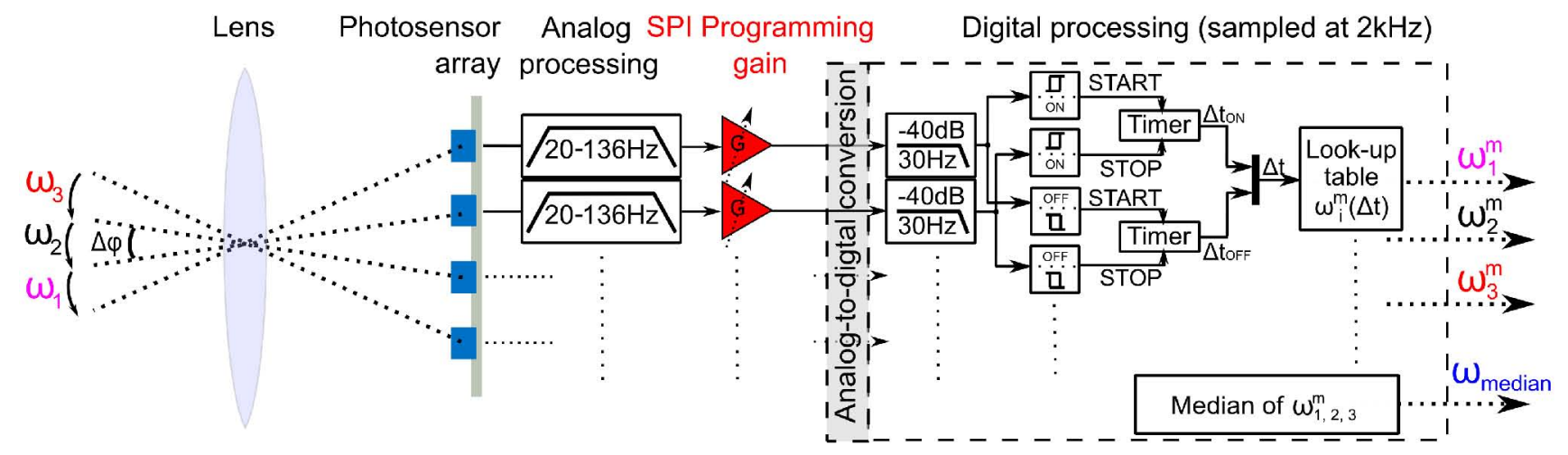

Fig. 1. Processing architecture of the new programmable-gain visual motion sensor. The 4 visual signals are filtered through an analog band-pass filter and amplified using a digital potentiometer. Then, they are filtered through a second order fixed-point digital low-pass filter. The 4 neighboring visual signals are then thresholded. In parallel, the time lag $\Delta t_{i}$ between each pair of signals is measured by a timer with a separation between ON (dark to light) and OFF (light to dark) contrasts, as previously found to occur in insects [21]. These $\Delta t_{i}$ are used to compute the 1-D angular speed $\omega_{i}^{m}$ in the visual field of each 1-D local motion unit. Lastly, the 3 local motion units are combined to generate a robust and frequently refreshed 1-D median angular speed, $\omega_{\text {median }}[25]$.

enabled a miniature helicopter to perform challenging tasks such as takeoff, terrain following, reacting suitably to wind, and landing. Unlike most of the autopilots classically used in manned helicopters, the OCTAVE autopilot was not designed to provide the aerial robot with height holding or ground speed holding skills. It simply ensured that "OF holding" occured so that the MAV would reach a "safe height" automatically at any ground speed. The main advantage of the OCTAVE autopilot was that it ensured that all manoeuvers and responses to disturbances (such as changes in terrain relief and demanding wind conditions) would occur at all costs without colliding with the ground: the result was measured in terms of task performances, and not in terms of the metric variables (airspeed, height, descent speed, etc.) usually monitored on-board most aircraft. The robot was able to takeoff, follow a shallow terrain, land and react to wind disturbances although it was completely unaware of parameters such as its airspeed, groundspeed, descent speed, its descent angle, its height over the terrain and its absolute altitude at any time. The OCTAVE robot acting upon the lift to hold the ventral OF constant accounts for many unexplained findings published during the last 70 years on insects' visually guided performances; for example, it accounts for the fact that honeybees descend in a headwind, land with a constant slope, and drown when travelling over mirror-smooth water [7].

The previous OCTAVE robot used a particular property of the optic flow when flying in pure translation along the $\mathrm{x}$-axis over an unknown terrain.

This movement generates an elementary translational OF, $\omega_{\Phi}$, which depends on the following equation:

$$
\omega_{\Phi} \sim \frac{v_{x}}{D} \cdot \sin (\psi+\Phi)
$$

where $v_{x}$ is the speed (ground speed) of the aircraft with respect to the ground, D is its distance from the ground, $\psi$ is the angle between the aircraft's speed vector and the horizon and $\Phi$ is the angle between the gaze direction and

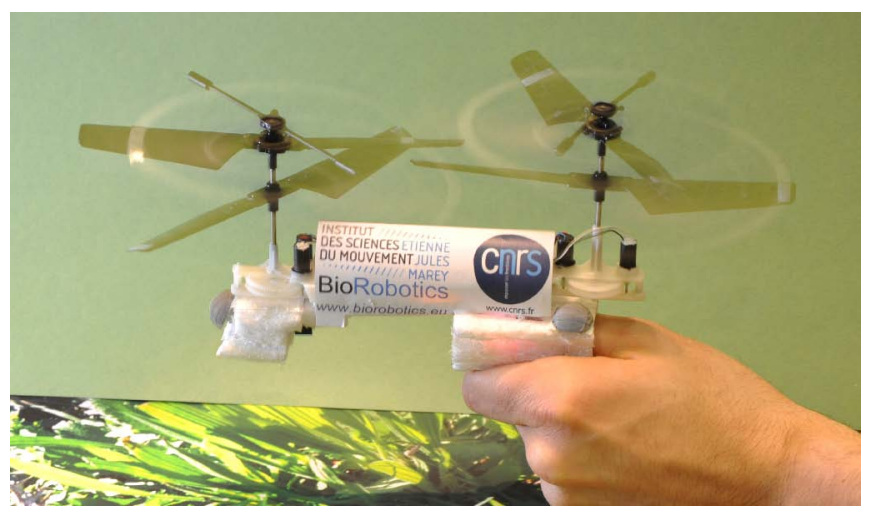

Fig. 2. TwinCoAx photograph showing the miniature free-flying helicopter equipped with a Bluetooth module and a programmable-gain visual motion sensor.

the horizon.

The eye of the previous OCTAVE helicopter was kept oriented downwards $\left(\Phi=90^{\circ}\right)$ so that $\mathrm{D}$ became the local height $\mathrm{h}$ with respect to the ground. Under these conditions, the OF $\omega_{90^{\circ}}$ depended simply on the ratio between the ground speed $v_{x}$ and the height h:

$$
\omega_{90^{\circ}} \sim \frac{v_{x}}{h}
$$

Previously, the use of a tethered aircraft was essential here to be able to implement and test the basic strategy used: OF regulation on an elementary rotorcraft with only three degrees of freedom. Quantitative tests on free-flying MAVs are more difficult to carry out and lack reproducibility because a MAV is unlikely to fly twice at the same height over the same site under the same wind disturbance conditions. In our quantitative tests, the whirling arm also introduced undesirable inertia into the control loop, which adversely affected both the heave and the surge dynamics: the previous OCTAVE robot was less agile than it would have been if it were flying freely. In this previous step, a supporting tether facilitated the parametric monitoring and the accessibility 


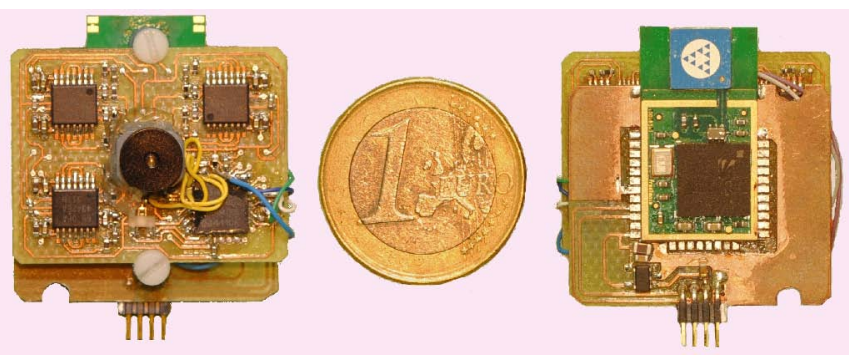

Fig. 3. Top view and bottom view of the electonic-board assembly composed of a 2-gram programmable-gain visual motion sensor (size: $23 \times 30 \mathrm{~mm}$ ) with its lens mounted on the photosensor array and a 1.7gram low-power Bluetooth module soldered onto its own PCB (thickness: $0.4 \mathrm{~mm})$.

and tuning of the perception/action loop, while making the experiments highly reproducible. We had therefore decided to use a step-by-step approach, starting with a tethered vehicle to test the fundamentals of the OCTAVE principle.

In the present paper, we describe how a programmable visual motion sensor and a VICON motion capture system made us advance one step further toward implementing optic flow regulation onboard a miniature free-flying helicopter.

\section{PRogrammable-Gain Visual Motion SENSOR INCORPORATED INTO THE MINIATURE FREE-FLYING TwinCoAx}

A miniature free-flying helicopter named TwinCoAx consisting of two co-axial rotary wings (see figure 2) was equipped with a Bluetooth link and a visual motion sensor. The TwinCoAx weighed less than 50 grams in all. The miniature free-flying helicopter was equipped with 3 InfraRed reflective markers so that the VICON system could record the helicopter's motion. The weight of the reflective markers was reduced by using only half a sphere in each case and by drilling a spherical hole in the back.

The electronic assembly mounted onboard the miniature free-flying helicopter (see figure 3) was composed of 2 modules:

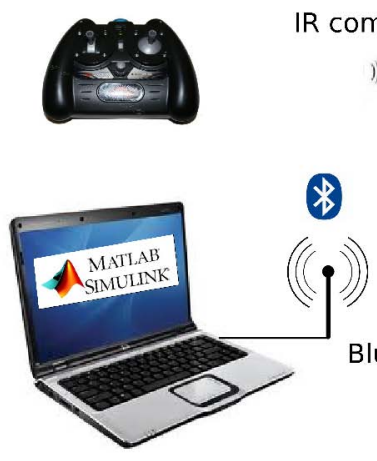

Ground computer

\section{R communication}

(i)) (i)) ) ()))

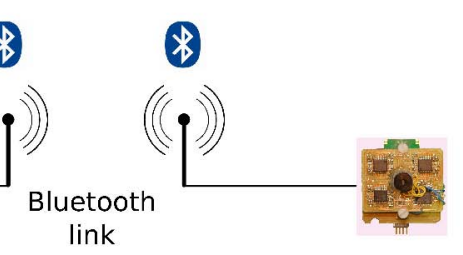

Embedded programmable Visual motion sensor

Fig. 4. Communication scheme between the ground and the miniature free-flying helicopter, named TwinCoAx. The free-flying helicopter was radiocontrolled manually using a conventional IR link. A wireless Bluetooth link was used to monitor the visual data back to the ground-based laptop via Matlab/Simulink.
- a new programmable-gain visual motion sensor (mass: 2 grams) inspired by the fly's eye,

- a tiny bluetooth module (module ALA from freetomove) emulating a RS232 serial link between the visual motion sensor and the ground.

The front end of the bio-inspired motion sensor was based on an off-the-shelf photodiode array called LSC (a component available at iC-Haus, www.ichaus.de) consisting of a small array of pixels (sensitive surface: $300 \mu \mathrm{m} \times 1600 \mu \mathrm{m}$ ) with on-chip preamplification. In the present study, only a row of 4 pixels was used. The photosensors, combined with a miniature lens (a Sparkfun SEN-00637 lens, focal length $2 \mathrm{~mm}$, f-number 2.8 , borrowed from a mobile telephone camera lens) creating 4 optical axes separated by the interreceptor angle $\Delta \varphi$, were combined with a programmablegain photocurrent amplifier. The visual signals were then used to process the angular speed with the "time of travel" scheme including ON and OFF pathway separation [15], [23]-[25]. The visual signal from the 4 adjacent pixels were filtered spatially by defocusing the lens (Fig. 1) from the sensor in order to obtain pixels featuring a Gaussian angular sensitivity similar to that of the fly [26]. These 4 visual signals were then amplified by a programmable gain and filtered temporally by means of a band-pass filter and thresholded to determine the 3 angular speeds $\omega_{i}^{m}$, defined as the ratio between the constant inter-receptor angle $\Delta \varphi$ and the time $\Delta t_{i}$ elapsing between the first and second thresholded signals of each local motion unit (see figure 1). This processing system has been described in detail in [14]. All in all, we obtained a cheap, programmable, accurate visual motion sensor by combining a set of 3 neighboring 2-pixel motion sensors and computing the median $\omega_{\text {median }}$ of the 3 output values $\left(\omega_{1}^{m}, \omega_{2}^{m}, \omega_{3}^{m}\right)$ [25].

The whole processing of the 3 local motion units and the calculation of the median value were carried out on a dsPIC33FJ128GP802 microcontroller working at a sampling

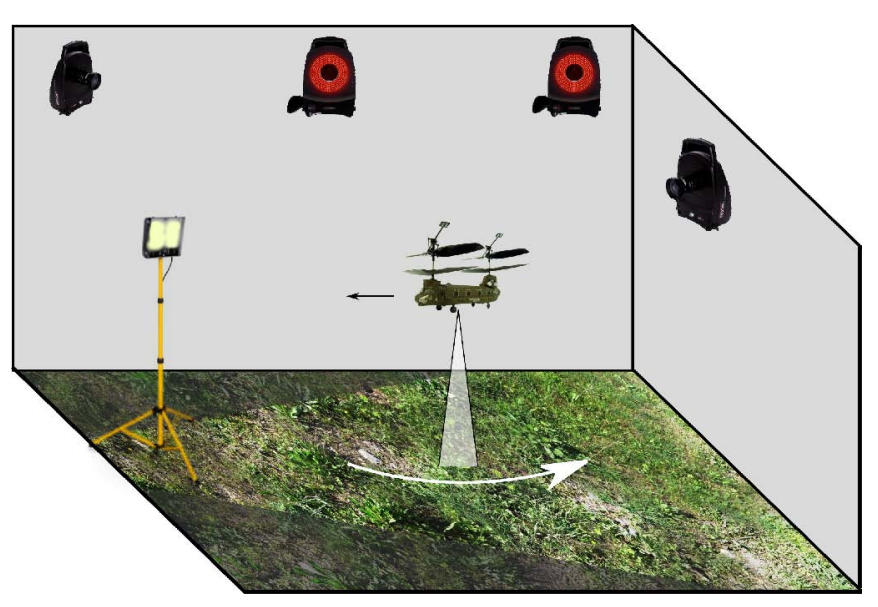

Fig. 5. The VICON-equipped free-flight facilities are composed of a ground covered with a natural pattern, a floodlight illuminating the scene in the visible domain and 6 VICON cameras to record the aircraft's motion. The floodlight was added because the background illuminance was initially only around 10lux in the VICON free-flying facilities. 

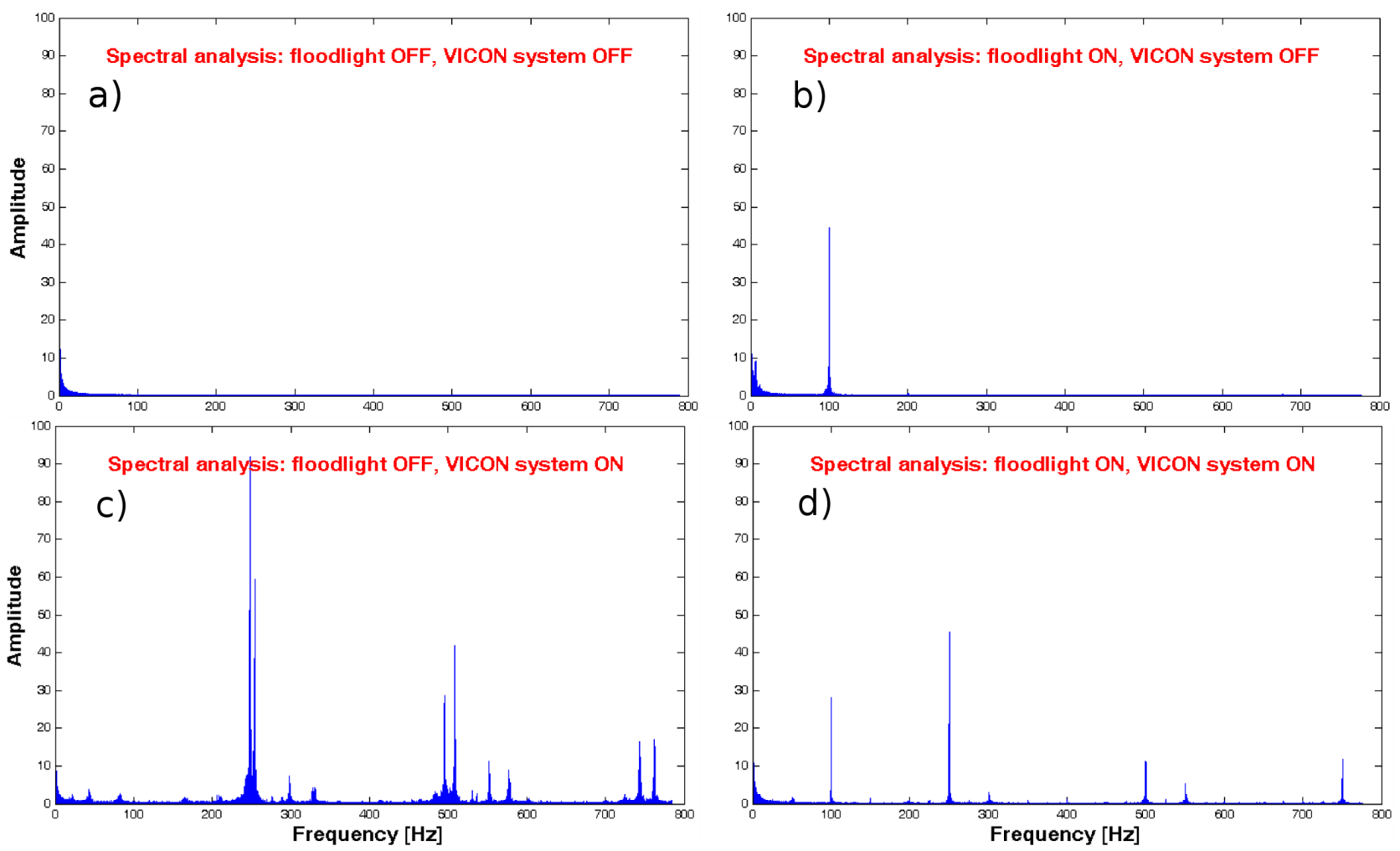

Fig. 6. Power spectrum of the $3 \mathrm{rd}$ photodiode of the photosensor array digitized at $1.6 \mathrm{kHz}$ under several lighting conditions. a) When the floodlight and the VICON system were switched OFF, the power spectrum was reduced to the low-frequency domain of the background visual scene. b) When the floodlight was ON and the VICON system was OFF, the visual spectrum included a single ray at $100 \mathrm{~Hz}$, corresponding to the 100-Hz lighting frequency of the floodlight. c) When the floodlight was OFF and the VICON system was ON, several powerful rays occurred at frequencies of about $250 \mathrm{~Hz}, 500 \mathrm{~Hz}$ and $750 \mathrm{~Hz}$, corresponding to the frequency of $250 \mathrm{~Hz}$ and the corresponding harmonics at which the infra-red LEDs in the VICON cameras illuminated the scene. d) When both the VICON camera system and the floodlight were switched ON, the visual spectrum showed several rays corresponding to the VICON camera and the floodlight power spectrums.

frequency of $2 \mathrm{kHz}$. The microcontroller incorporated in the visual motion sensor (Fig. 3) is connected to an external Bluetooth module, which conveys the data to a computer for analysis.

The communication scheme between the ground and the miniature helicopter can be seen in figure 4. The helicopter's trajectory was controlled manually using an IR remote control borrowed from a conventional model aircraft; the data from the visual motion sensor were collected on a groundbased laptop via a wireless Bluetooth link.

\section{FREE-FLYING HELICOPTER LOCATED BY A VICON SYSTEM}

The free-flight facilities included (see figure 5):

- a ground printed with a natural scene,

- a VICON system recording the motion of the 50-g helicopter, and

- a floodlight illuminating the visual scene.

The lighting of the free-flight facilities was therefore fairly complex, since it consisted of both the floodlight and the Infra-red pulses used by the VICON system to track the free-flying robot. This complex visual scene was studied by recording the output signal emitted by one of the photodiodes in our visual motion sensor under various lighting conditions.
Figure 6 shows the contribution made by the VICON camera system to the visual scene by adding high-energy rays in the frequency domain. Since the photodiodes of the LSC sensor are sensitive in the visible range as well as the near infrared spectral range, our visual motion sensor was sensitive to the high-energy infra-red pulses emitted by the VICON system. To attenuate these high-frequency perturbations that disturbed the visual signals, we increased from 4th to 8 th the order of magnitude of the fixed-point low pass filter (see figure 1).

\section{Visual MOTION MEASUREMENTS OBTAINED DESPITE THE VICON LIGHTING PERTURBATIONS}

Figure 7 shows the indoor dynamic responses of the programmable-gain visual motion sensor mounted onboard a free-flying miniature helicopter. Although the eye of the freeflying helicopter was not kept exactly oriented downwards at all times because of the slight helicopter pitch, and although the pitch variations may have added some rotational optic flow, the ground-truth optic flow can be modelled by the following equation:

$$
\omega_{\text {ground-truth }} \text { 90 }^{\circ}=\frac{v_{x}}{h}
$$

Despite the complexity of the visual scene due to the 
a)

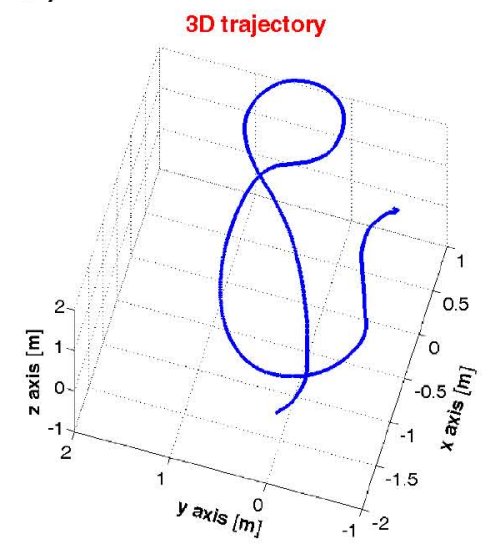

II

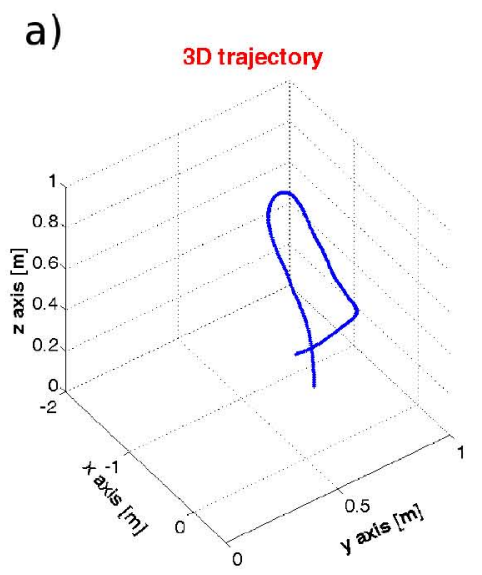

Projected trajectory in the xy plane
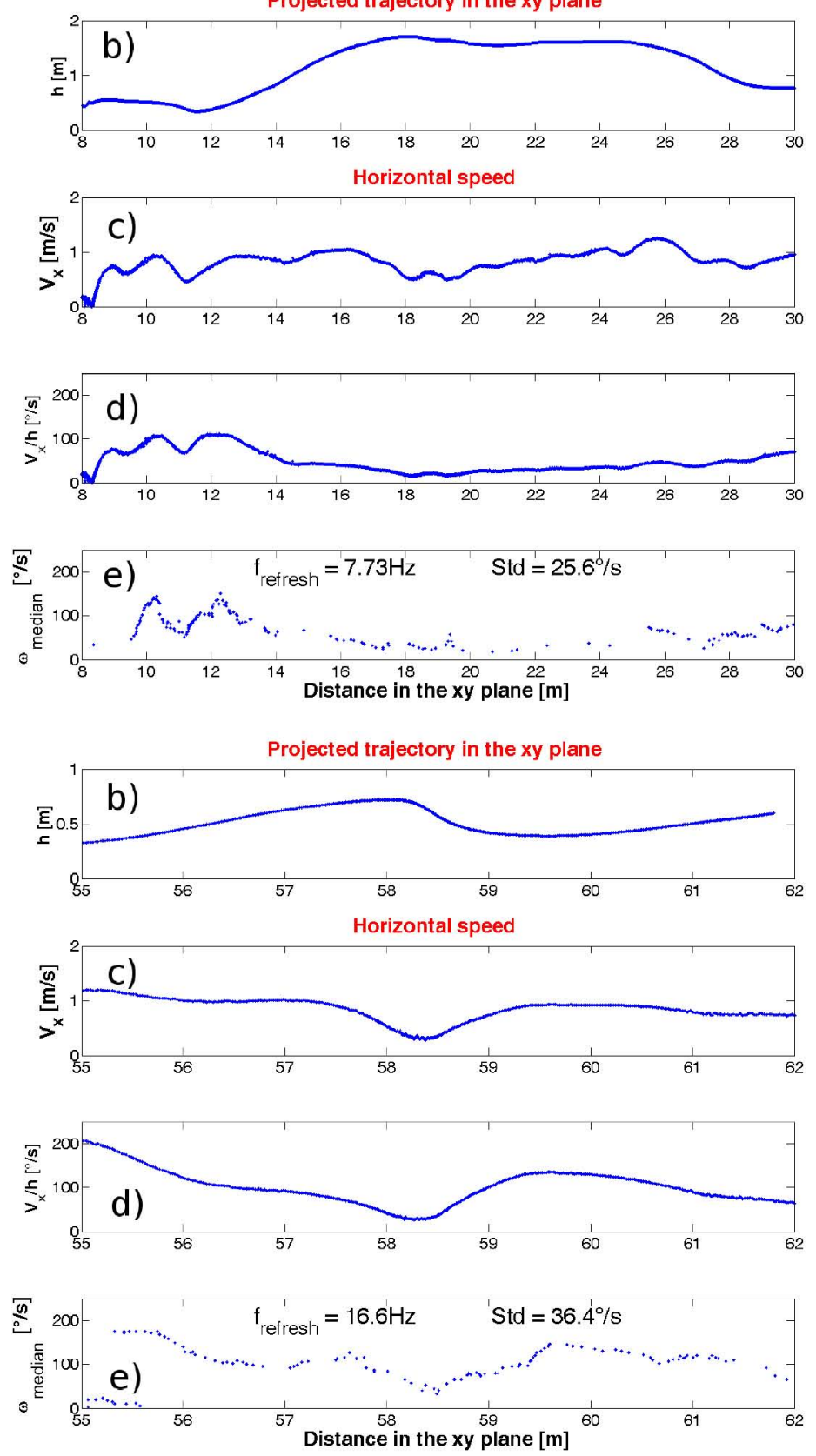

Fig. 7. Two examples of trajectories showing the accuracy of the visual angular velocity measurements performed onboard the helicopter. Despite the complex trajectory, the complex pattern of illuminance and the fact that the natural visual environment was devoid of highly contrasting patterns, the response of the visual motion sensor was remarkably accurate, showing a dispersion of less than $40^{\circ} / \mathrm{s}$, and the refresh rate makes it highly suitable for robotic applications. I-II a) 3-D trajectory of the miniature free-flying helicopter I-II b) Trajectory of the miniature free-flying helicopter projected in the x-y plane (the vertical plane). I-II c) $\frac{V_{x}}{h}$ : Approximate ground-truth optic flow generated by the free-flying helicopter. I-II d) Visual angular velocities measured onboard the free-flying helicopter using the programmable-gain visual motion sensor. The refresh rate $f_{\text {refresh }}$ (the number of new measurements per second) and the standard deviation of the error between the theoretical value of the optic flow $\left(\frac{V_{x}}{h}\right)$ and the measured optic flow $\omega_{m e d i a n}$ are indicated in the figure.

infra-red perturbations introduced by the VICON camera, the visual motion sensor responded accurately when flying over a ground covered by a natural printed pattern (Fig 7 I-II e). The standard deviation of the error between the ground-truth value of the optic flow $\left(\frac{V_{x}}{h}\right)$ and the measured optic flow $\omega_{\text {median }}$ was lower than $40^{\circ} / \mathrm{s}$, which is quite low. The refresh rate of the sensor $f_{\text {refresh }}$ (the number of new measurements per second) was greater than $7 \mathrm{~Hz}$, which makes this sensor highly suitable for robotic applications.

When mounted on the helicopter, the visual motion sen- 
sor was subjected to the strong $250-\mathrm{Hz}$ Infra-Red pulses produced by the VICON system as well as the helicopter vibrations. The robust performances observed despite these disturbances show that this sensor is suitable for performing optic flow regulation by including it in a direct feedback loop.

\section{CONCLUSION}

The performances of a visual motion sensor mounted onboard a miniature helicopter for measuring the local 1$\mathrm{D}$ angular speed were compared here for the first time with the ground-truth optic flow approximated using a VICON motion capture system.

The ambient indoor illuminance was closely analyzed and the visual motion processing was adjusted to cope with the complex lighting conditions introduced by the VICON system.

In addition to its robustness to strong illuminance variations [14], the present bio-inspired 4-pixel motion sensor equipped with the "time of travel" scheme proved to be a highly accurate, quasi-linear, quickly refreshed motion sensor under real flying conditions, and its performances confirmed that it is suitable for use on free-flying robots. The new electronic board assembly now being developed will shortly be mounted on the TwinCoAX robot for performing straight flight and optic flow regulation onboard, and in particular to confirm the landing and terrain following abilities of aircraft equipped with this sensor, which functions efficiently without requiring any information about the velocity or the altitude.

In conclusion, this study brings us one step further toward the goal of implementing visual motion sensors on lightweight miniature autonomous aerial robots.

\section{ACKNOWLEDGMENT}

We are most grateful to B.-J. Fuchslock and M. Boyron for their assistance with the electronic design, J. Diperi for the mechanical design, P. Berthe, G. Rao, T. Coyle and E. Berton for their kind help with the VICON system, J. Blanc for improving the English manuscript and N. Franceschini and S. Viollet for their fruitful comments and suggestions during this research.

\section{REFERENCES}

[1] N. Franceschini, J. M. Pichon, and C. Blanes, "From insect vision to robot vision," Philosophical Transactions of the Royal Society B: Biological Sciences, vol. 337, pp. 283-294, 1992.

[2] G. L. Barrows, C. Neely, and K. T. Miller, "Optic flow sensors for mav navigation," in Fixed and Flapping Wing Aerodynamics for Micro Air Vehicle Applications, AIAA, Ed., vol. 191, Progress in Astronautics and Aeronautics, 2001, pp. 557-574.

[3] F. Ruffier and N. Franceschini, "Octave, a bioinspired visuo-motor control system for the guidance of micro-air vehicles," in Proc. of the Conference on Bioengineered and Bioinspired Systems, A. RodriguezVazquez, D. Abbott, and R. Carmona, Eds., vol. SPIE 5119. SPIE Bellingham USA, 2003, pp. 1-12.

[4] - "Optic flow regulation: the key to aircraft automatic guidance," Robotics and Autonomous Systems, vol. 50, no. 4, pp. 177 - 194, 2005.

[5] S. Griffiths, J. Saunders, A. Curtis, B. Barber, T. McLain, and R. Beard, "Maximizing miniature aerial vehicles," Robotics \& Automation Magazine, IEEE, vol. 13, pp. 34-43, 2006.
[6] J.-C. Zufferey and D. Floreano, "Fly-inspired visual steering of ultralight indoor aircraft," IEEE Transactions on Robotics, vol. 22(1), pp. 137-146, 2006.

[7] N. Franceschini, F. Ruffier, and J. Serres, "A bio-inspired flying robot sheds light on insect piloting abilities," Current Biology, vol. 17, pp. $329-335,2007$.

[8] M. Garratt and J. Chahl, "Vision-based terrain following for an unmanned rotorcraft," Journal of Field Robotics, vol. 25, pp. 284301, 2008.

[9] J. Conroy, G. Gremillion, B. Ranganathan, and J. Humbert, "Implementation of wide-field integration of optic flow for autonomous quadrotor navigation," Autonomous robots, vol. 27, no. 3, pp. 89-198, 2009.

[10] A. Beyeler, J. C. Zufferey, and D. Floreano, "Vision-based control of near-obstacle flight," Autonomous robots, vol. 27, pp. 201-219, 2009.

[11] B. Herisse, T. Hamel, R. Mahony, and F.-X. Russotto, "The landing problem of a vtol unmanned aerial vehicle on a moving platform using optical flow," in Proc. of International Conference on Intelligent Robots and Systems (IROS), 2010 IEEE/RSJ, 2010, pp. 1600 - 1605.

[12] F. Kendoul, Z. Yu, and K. Nonami, "Guidance and nonlinear control system for autonomous flight of minirotorcraft unmanned aerial vehicles," Journal of Field Robotics, vol. 27(3), pp. 311-334, 2010.

[13] S. Fuller and R. Murray, "A hovercraft robot that uses insect-inspired visual autocorrelation for motion control in a corridor," in IEEE Conference on Robotics and Biomimetics (ROBIO), Phuket, Thailand, 2011, pp. 1474-1481.

[14] F. Expert, S. Viollet, and F. Ruffier, "Outdoor field performances of insect-based visual motion sensors," Journal of Field Robotics, vol. 28, no. 4, pp. 529-541, 2011.

[15] C. Blanes, Appareil visuel elementaire pour la navigation a vue d'un robot mobile autonome. Marseille: Master thesis in Neurosciences (DEA in French),Advisor: N. Franceschini, Neurosciences, Univ. AixMarseille II, 1986.

[16] J. M. Pichon, C. Blanes, and N. Franceschini, "Visual guidance of a mobile robot equipped with a network of self-motion sensors," in Mobile Robots IV, vol. Vol. 1195. SPIE Bellingham USA, 1989, pp. 44-53.

[17] S. Viollet and N. Franceschini, "Super-accurate visual control of an aerial minirobot," In Autonomous minirobots for research and edutainment (AMIRE), vol. 1, pp. 215-224, 2001.

[18] S. Viollet, F. Ruffier, T. Ray, M. Menouni, F. Aubépart, L. Kerhuel, and N. Franceschini, "Characteristics of three miniature bio-inspired optic flow sensors in natural environments," in Proc. of 2010 Fourth International Conference on Sensor Technologies and Applications (SENSORCOMM), 2010, pp. 51 - 55.

[19] F. Expert, S. Viollet, and F. Ruffier, "A mouse sensor and a 2 pixel motion sensor exposed to continuous illuminance changes," in IEEE Sensors 2011 conference, Limerick, Ireland, 2011, pp. 974-977.

[20] F. G. Bermudez and R. Fearing, "Optical flow on a flapping wing robot," in IEEE International Conference on Intelligent Robots and Systems (IROS), 2009, pp. 5027 - 5032.

[21] N. Franceschini, A. Riehle, and A. L. Nestour, Facets of vision. Springer, Berlin, 1989, ch. 17, Directionally Selective Motion Detection by Insect Neurons, pp. 360-390.

[22] F. Ruffier and N. Franceschini, "Visually guided micro-aerial vehicle : automatic take off, terrain following, landing and wind reaction," in Proceeding of IEEE International Conference on Robotics and Automation (ICRA 2004), New Orleans, USA, 2004, pp. 2339-2346.

[23] F. Ruffier, S. Viollet, S. Amic, and N. Franceschini, "Bio-inspired optical flow circuits for the visual guidance of micro-air vehicles," Proceeding of IEEE International Symposium on Circuits and Systems (ISCAS), vol. 3, pp. 846-849, 2003.

[24] N. Franceschini, F. Ruffier, J. Serres, and S. Viollet, Aerial vehicles. Vienna : In-Tech, Eds : T. M. Lam, 2009, ch. 35 : Optic flow based visual guidance: from flying insects to miniature aerial vehicles, pp. $747-770$.

[25] F. Roubieu, F. Expert, M. Boyron, B.-J. Fuschlock, S. Viollet, and F. Ruffier, "A novel 1-gram insect based device measuring visual motion along 5 optical directions," in IEEE Sensors 2011 conference, Limerick, Ireland, 2011, pp. 687-690.

[26] K. Götz, "Optomotorische Untersuchung des visuellen systems einiger Augenmutanten der Fruchtfliege Drosophila," Biological Cybernetics, vol. 2, pp. 77-92, 1964. 